\title{
Preliminary Results on Irradiance Measurements from Lyra and Swap
}

\author{
S. T. Kumara, ${ }^{1}$ R. Kariyappa, ${ }^{2}$ M. Dominique, ${ }^{3}$ D. Berghmans, ${ }^{3}$ L. Damé, ${ }^{4}$ J. F. Hochedez, ${ }^{4}$ \\ V. H. Doddamani, ${ }^{5}$ and Lakshmi Pradeep Chitta ${ }^{2}$ \\ ${ }^{1}$ Department of Physics, Auden Technology and Management Academy (ATMA), Bangalore 562112, India \\ ${ }^{2}$ Indian Institute of Astrophysics, Bangalore 560 034, India \\ ${ }^{3}$ Royal Observatory of Belgium (ROB), Circular Avenue 3, B-1180 Brussels, Belgium \\ ${ }^{4}$ LATMOS, 11 Boulevard d'Alembert, 78280 Guyancourt, France \\ ${ }^{5}$ Department of Physics, Bangalore University, Bangalore 560056, India
}

Correspondence should be addressed to S. T. Kumara, kumarapsce@rediffmail.com

Received 14 November 2011; Accepted 9 December 2011

Academic Editor: J. Javaraiah

Copyright () 2012 S. T. Kumara et al. This is an open access article distributed under the Creative Commons Attribution License, which permits unrestricted use, distribution, and reproduction in any medium, provided the original work is properly cited.

\begin{abstract}
The first and preliminary results of the photometry of Large Yield Radiometer (LYRA) and Sun Watcher using Active Pixel system detector and Image Processing (SWAP) onboard PROBA2 are presented in this paper. To study the day-to-day variations of LYRA irradiance, we have compared the LYRA irradiance values (observed Sun as a star) measured in Aluminum filter channel (171 $\AA-$ $500 \AA$ ) with spatially resolved full-disk integrated intensity values measured with SWAP $(174 \AA)$ and Ca II K $1 \AA$ index values (ground-based observations from NSO/Sac Peak) for the period from 01 April 2010 to 15 Mar 2011. We found that there is a good correlation between these parameters. This indicates that the spatial resolution of SWAP complements the high temporal resolution of LYRA. Hence SWAP can be considered as an additional radiometric channel. Also the K emission index is the integrated intensity (or flux) over a $1 \AA$ band centered on the $\mathrm{K}$ line and is proportional to the total emission from the chromosphere; this comparison clearly explains that the LYRA irradiance variations are due to the various magnetic features, which are contributing significantly. In addition to this we have made an attempt to segregate coronal features from full-disk SWAP images. This will help to understand and determine the actual contribution of the individual coronal feature to LYRA irradiance variations.
\end{abstract}

\section{Introduction}

The Sun is the primary source of energy responsible for governing both the weather and climate of Earth. For that reason alone one would expect that changes in the amount and type of energy Earth received from the Sun could alter weather and climate on the Earth. Hence the knowledge of the solar spectral irradiance is of large interest to solar physics, aeronomy, and to other fields of heliospheric or planetary research. The solar ultraviolet (UV) irradiance below $3000 \AA$ is the main source of the energy converted in the Earth's atmosphere, controlling its thermal structure, dynamics, and chemistry through photodissociation and photoionization [1]. Because of these, changes in UV irradiance influence the concentration of the ozone in the Earth's atmosphere [2, 3 ] and may play a significant role in the process of the global warming. The balance of the ozone formed by radiation below $2400 \AA$ in the stratosphere and mesosphere is of special interest for life and mankind. However, the photons above $2000 \AA$ get dissociated in the stratosphere and disturb the balance of the ozone (e.g., $[4,5])$. This is because the ozone gas is produced naturally in the stratosphere where it strongly absorbs incoming UV radiation. But as stratospheric ozone decreases, UV radiation is allowed to pass through, and exposure at the Earths surface increases. Exposure to shorter wavelengths increases by a larger percentage than exposure to longer wavelengths. The UV irradiance variability has significant effects on human technologies too, currently addressed in the frame of Space Weather studies.

For more than two decades, solar UV irradiance observations have been monitored from several satellites. These space UV irradiance observations have shown that the UV flux changes over the solar cycle, being higher during 
maximum activity conditions, and short-term changes (from days to months) are superposed on the long-term variation $[6,7]$. The long-term UV irradiance variations are attributed to the changing emission of bright magnetic elements [810], and the short-term variations are directly associated with the growth and decay of active regions [11]. The current UV irradiance models are based on integrated full-disk surrogates; therefore they cannot provide adequate information on the physical causes of the observed UV irradiance changes. In the last decade, there are good time sequence observations available from several space experiments, Solar Heliospheric Observatory/Extreme Ultraviolet Imaging Telescope/Michelson Doppler Imager (SOHO/EIT/ $\mathrm{MDI}$ ), to measure the various parameters from spatially resolved images of the Sun. And recently we have highresolution observations from Solar Dynamic Observatory/ Atmospheric Imaging Array/Helioseismic-Magnetic Imager (SDO/AIA/HMI), Project for Onboard Autonomy 2/Large Yield Radiometer/Sun Watcher using Active Pixel system detector and Image Processing (PROBA2/LYRA/SWAP). All of them differ in spectral coverage, time coverage, time cadence, and nature of the instrument (spectrograph, photometer, or imager). In addition, we have ground-based observations of Ca II K spectroheliograms from NSO/Sac Peak and also from many other observatories in the world. All these observations will provide the best data set so far. The strategy is thus to bridge the most reliable observations, with the best possible models (e.g., [12-14]). The spatially resolved full-disk images obtained from space have to be compared with solar images from ground-based observations. The full-disk precise photometric images and full-disk magnetograms will help to explore the temporal and spatial variability of the solar UV irradiance and to determine the couplings with the magnetic structures.

PROBA2 is the second satellite in the European Space Agency's series of PROBA low-cost satellites that are being used to validate new spacecraft technologies while also carrying scientific instruments. It was launched on November 2, 2009, with a Rockot launcher in a sun-synchronous low Earth orbit at an altitude of $725 \mathrm{~km}$. It provides a technology demonstration platform for testing a number of instruments and techniques relevant to solar physics, space weather, aeronomy, avionics, spacecraft attitude control, power system, and propulsion. The orbit of PROBA2 is eclipse-free for nine months per year, thus the orbit is well suited for the solar observing instruments onboard, namely, LYRA and SWAP.

LYRA is the solar UV radiometer that will embark in 2006 onboard PROBA2, a technologically oriented ESA micromission $[15,16]$. It will be monitoring the solar irradiance in four carefully selected UV passbands. The channels have been chosen for their relevance to solar physics, aeronomy, and space weather Figure 1: (1) the 1150-1250 ̊ Lyman- $\alpha$, (2) the 2000-2200 $\AA$ Herzberg continuum range, (3) the Aluminium filter channel (171-500 $\mathrm{A})$ including He II at $304 \AA$, and (4) the Zirconium filter channel (10-200 $)$. LYRA benefited from wide bandgap detectors based on diamond. It was the first space assessment of a pioneering UV detectors program. It makes the sensors radiation hard and "solar-blind", which makes dispensable filters that block the

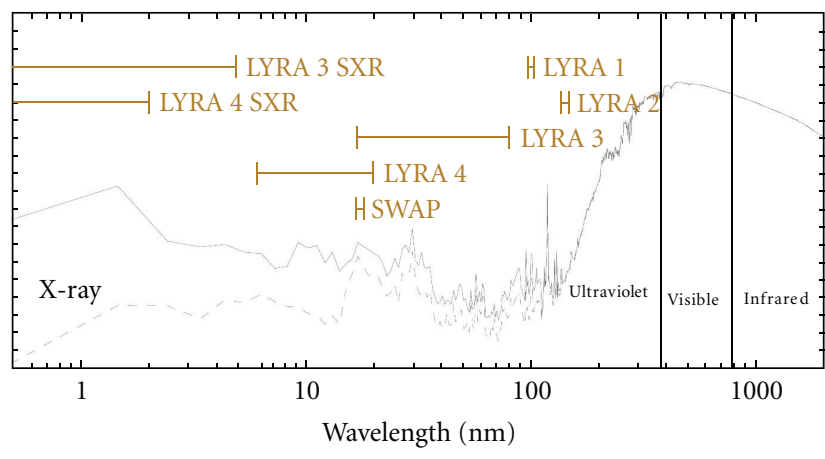

FIgure 1: The bandpasses of SWAP and four LYRA channels over two solar spectra, one from solar maximum and one from solar minimum, observed by Thermosphere Ionosphere Mesosphere Energetics and Dynamics Mission/Solar EUV Experiment (TIMED/SEE) and Solar Radiation and Climate Experiment (SORCE) (Courtesy: Dr. Sarp Yalim).

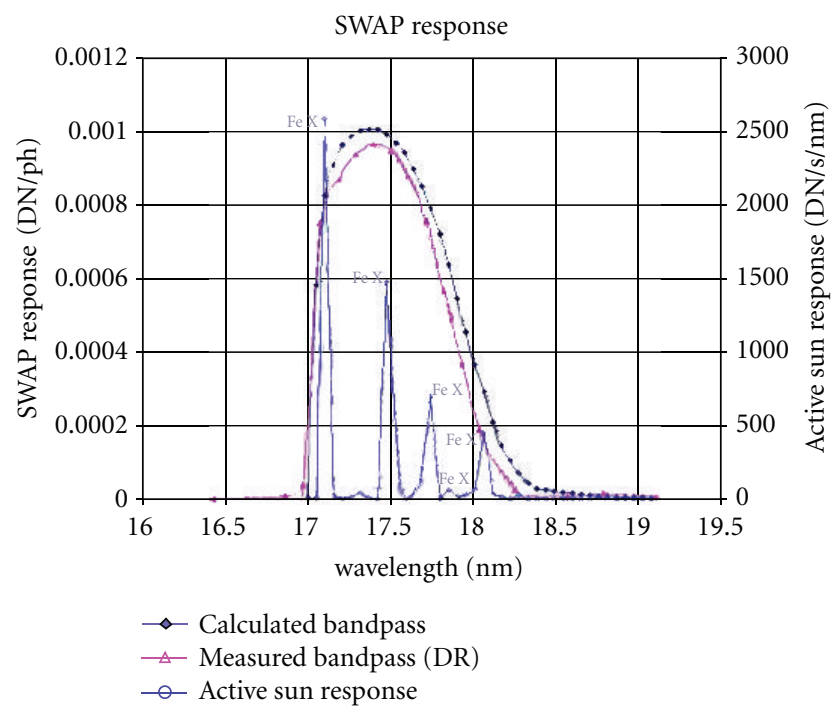

FIGURE 2: The bandpass of SWAP with its nominal spectral interval with peak at $17.4 \mathrm{~nm}$ (Courtesy: Dr. Sarp Yalim).

unwanted visible, but attenuate seriously the desired UV radiation. LYRA has a very high cadence up to $100 \mathrm{~Hz}$.

SWAP $[17,18]$ telescope is a compact EUV imager on board the PROBA2 mission that will observe the Sun in extreme ultraviolet (EUV) and provides continuous images of the Sun (solar corona) in a narrow bandpass with peak at $174 \AA$ (Figure 2), corresponding to a formation temperature of 1 million degree Kelvin. The SWAP instrument was built upon the heritage of the Extreme ultraviolet Imaging Telescope (EIT) which monitors the solar corona since 1996 on board the SOHO mission. SWAP has a field of view of 54 arcmin. This field of view can easily be extended though spacecraft offpointings that can be commanded from the PROBA2 Science Center in a matter of hours. The effective field of view for static coronal structures is therefore 65 arcmin and probably even larger for eruptions. This is much larger than the FOV of SOHO/EIT ( 45 arcmin) or 
SDO/AIA (41 arcmin). Whereas the AIA imager on SDO will provide unprecedented images in spatial and temporal resolution of the solar disk, SWAP is the only EUV imager in space to fill the gap between the solar limb and the farout corona as imaged by the SOHO/LASCO (Large Angle and Spectrometric Coronagraph) coronagraphs. SWAP also offers the advantage of high image cadence (maximal 3 per minute, 1 per minute in nominal operations) to monitor transitory phenomena. With this higher cadence, SWAP will monitor events in the low solar corona that might be relevant for space weather. These events include EIT waves (global waves propagating across the solar disc from the CME eruption site), EUV dimming regions (transient coronal holes from where the CME has lifted off), and filament instabilities (a specific type of flickering during the rise of a filament).

The $\mathrm{H}$ and $\mathrm{K}$ lines of ionized calcium have been recognized as useful indicators for identifying regions of chromospheric activity on the solar surface. These lines are very sensitive to the variations in temperature and the magnetic field strength. Therefore they are excellent indicators of the chromospheric structural changes related to solar magnetic activity. The images observed in $\mathrm{Ca}$ II $\mathrm{H}$ or $\mathrm{K}$ line and in He II line are identical to each other. The parameter that best quantifies the chromospheric emission in the $\mathrm{K}$ line is the so-called $\mathrm{K}$ emission index introduced by [19]. The $\mathrm{K}$ emission index is the integrated intensity (or flux) over a $1 \AA$ band centered on the $\mathrm{K}$ line and is proportional to the total emission from the chromosphere [9,20-25]. The Sun viewed as a star through a $1 \AA$ passband filter centered on the $\mathrm{K}$ line would appear as a variable, showing both the rotational modulation and the 11-year cycle [26].

\section{Observations and Data Analysis}

In this paper, LYRA irradiance observations measured in channel 3 (Aluminium filter channel: $171 \AA-500 \AA$, including He II at $304 \AA$ ), SWAP (174 $\AA$ ) full-disk integrated intensity (SWAVINT, level-3) values, and Ca II K (393.4 nm) time series values (corresponding to $1 \AA$ emission index) for the period of observations from April 1, 2010 to March 15, 2011 are used. To determine the day-to-day variations of LYRA channel-3 (cadence averaged to 1 minute), SWAP (cadence averaged to 2 minute) time series, and Ca II K $1 \AA \AA$ emission index, we have read the fits files of LYRA and SWAP data and carried out the analysis using Solar Software (SSW) library in IDL. SWAVINT is a keyword in the calibrated SWAP fits files and corresponds to the average intensity of a whole SWAP image (i.e., a fully calibrated image except applying transformation, which is placing the Sun in the center of the image and aligning the solar north to the top of the image) normalized with its exposure time

$$
\text { SWAVINT }=\frac{1}{t} \frac{1}{P} \sum_{i=1}^{P} \mathrm{DN}_{i}
$$

where $t$ is the exposure time of the image, $P$ is the number of pixels which is $1024 \times 1024$ for the whole image, and DN is the digital number in pixel $i$. The unit of SWAVINT is DN/s. The dynamic range of SWAP is much smaller than the one

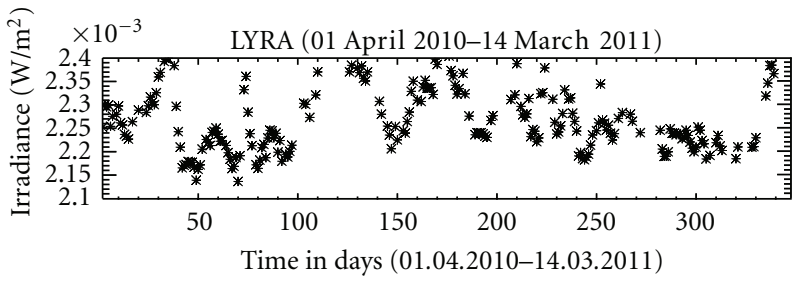

(a)

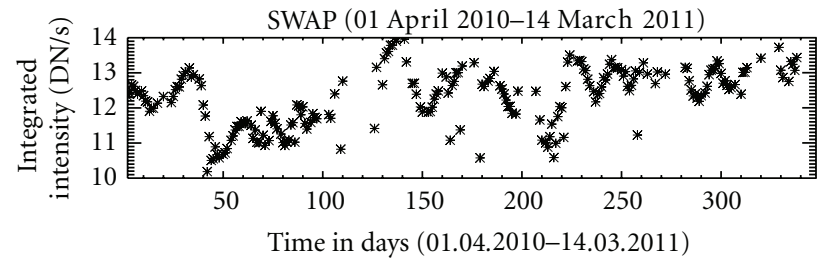

(b)

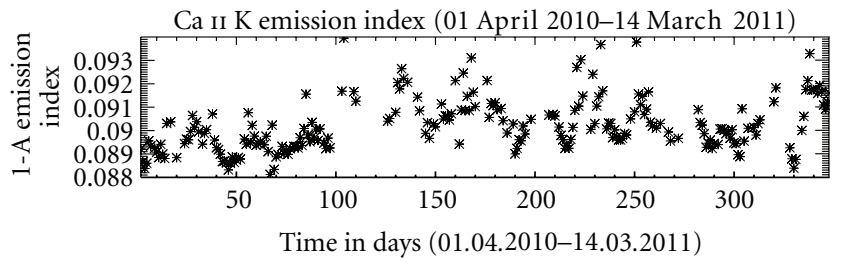

(c)

FIGURE 3: Time series (day-to-day variations) of LYRA irradiance values of channel 3 (171 $\AA-500 \AA)$, full-disk integrated intensity values of SWAP $(174 \AA)$, and $1 \AA$ emission index values of Ca II K $(393.4 \mathrm{~nm})$ for the period from 01 April 2010 to $15 \mathrm{Mar}$ 2011. Note that there is a good correlation in all the three time series.

of LYRA. SWAP is not designed to see flares at full extent. As a consequence of the limited range, flare pixels are soon saturated and so the flares can hardly be seen in SWAVINT since only a few pixels are affected and saturated immediately.

\section{Results and Discussion}

We have compared the LYRA irradiance values (observed Sun as a star) measured in channel 3 (Aluminium filter channel: $171 \AA-500 \AA$, which includes He II at $304 \AA$ also) with spatially resolved full-disk integrated intensity values measured in SWAP (174 $\AA$ ) for the period from 01 April 2010 to 15 Mar 2011. Also we have compared LYRA irradiance values and SWAP full-disk integrated intensity values with $1 \AA$ emission index values of Ca II K (ground based observations from $\mathrm{NSO} / \mathrm{Sac}$ Peak) for the same period. We have observed from the time series plot (Figure 3 ) and scatter plot (Figure 4) that the long-term variability of the LYRA channel 3 , the Aluminum filter channel has shown a good correlation with spatially resolved full-disk integrated intensity values of SWAP and the correlation is found to be 60 percent $(r=$ 0.6). However the correlation between LYRA and Ca II K $(r=0.47)$, as well as between SWAP and Ca II K $(r=$ $0.5)$, is comparatively lesser than the correlation between LYRA and SWAP. This is because there is a data gap in $\mathrm{Ca}$ II $\mathrm{K} 1 \AA$ emission index values. These results suggest that 


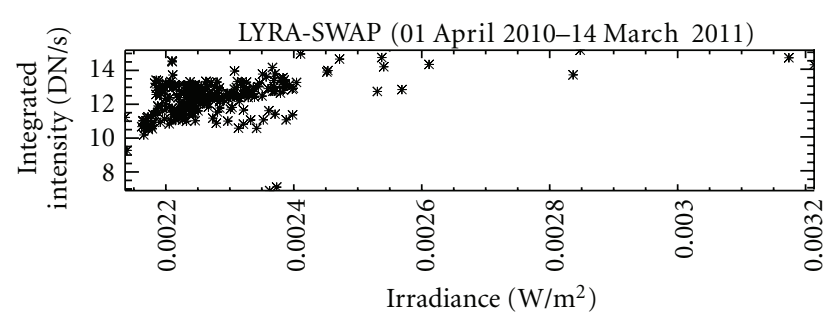

(a)

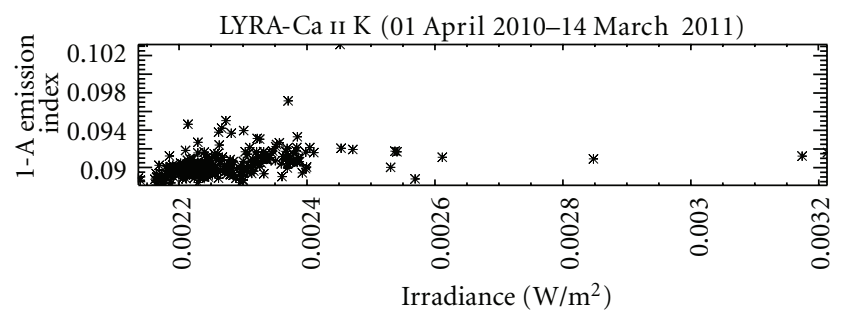

(b)

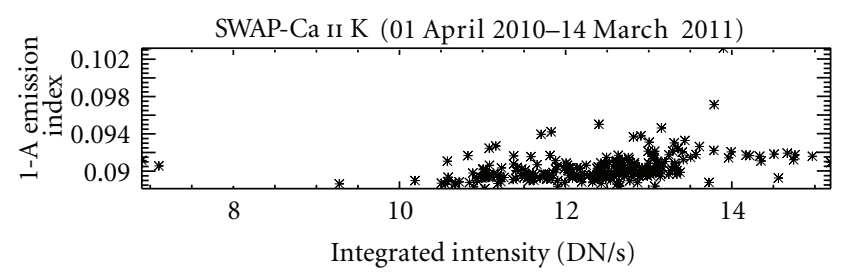

(c)

FIGURE 4: (a) Scatter plot between LYRA irradiance values of channel 3 and full-disk integrated intensity values of SWAP $(r=$ 0.6). (b) Scatter plot between LYRA irradiance values of channel 3 and $1 \AA$ emission Index values of Ca II K $(r=0.47)$. (c) Scatter plot between full-disk integrated intensity values of SWAP and $1 \AA$ emission Index values of Ca II K $(r=0.5)$.

the LYRA irradiance variations are due to the variations of solar magnetic features observed in SWAP and Ca II K. The spatial resolution of SWAP complements the high temporal resolution of LYRA. Hence SWAP can be considered as an additional radiometric channel. Further these comparisons clearly explain the irradiance variations are due to the various magnetic features, which are contributing significantly.

In order to understand the actual contribution of the individual coronal features to LYRA irradiance variations, we are segregating the coronal features from full-disk spatially resolved images obtained from PROBA2/SWAP (174 $\AA$ ). The morphological structures (pattern recognition), geometrical location, intensity levels and gradients, contrasts, and the sizes will be used as main criteria to recognize and segregate the various coronal features. We have done a very preliminary analysis on the SWAP images to segregate different coronal features. A sample of segregated image containing active regions is shown in Figure 5 in comparison with original image. Further detailed image analysis on large number of full-disk images is in progress. These results are helpful to make feature-to-feature correlations and to understand and establish their role and contribution to UV irradiance variability. Also these investigations will help better to understand

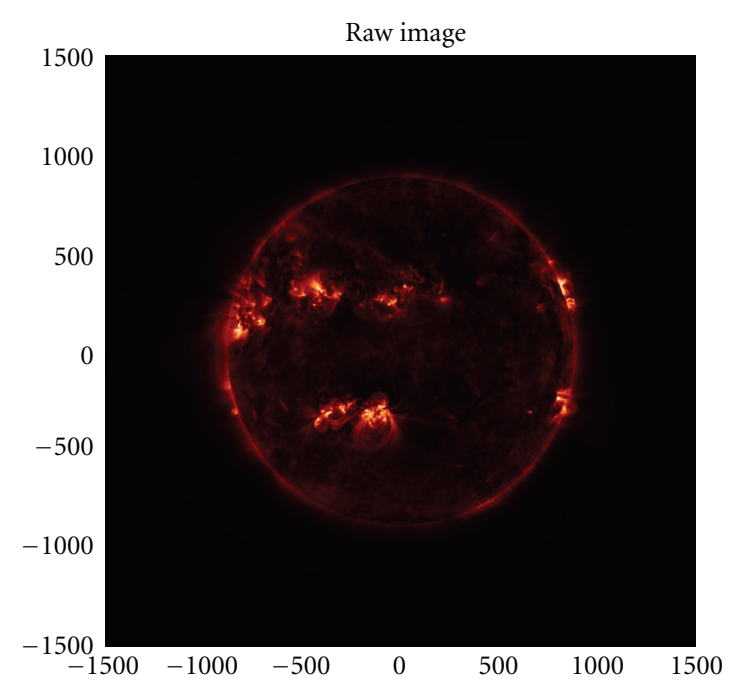

(a)

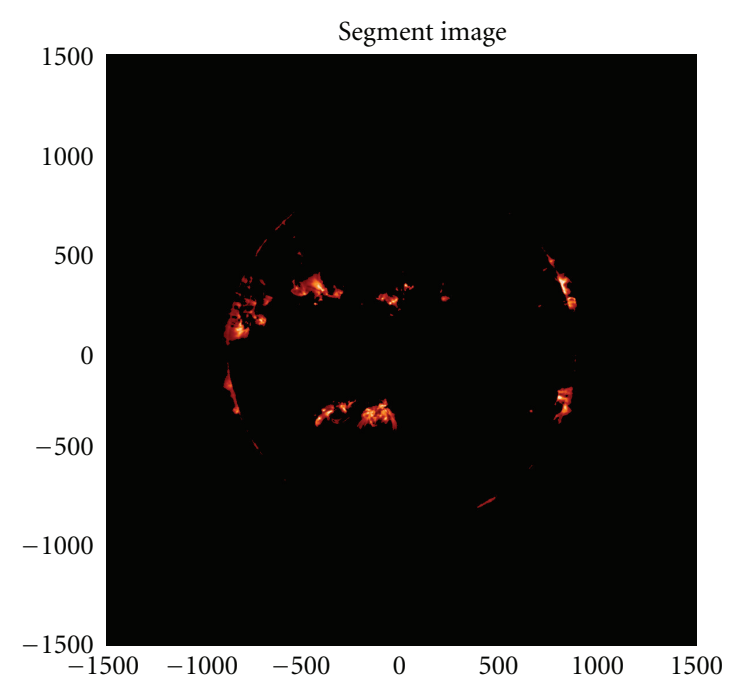

(b)

FIGURE 5: (a) Represents original SWAP image and (b) segregated (only active regions) SWAP image.

the nature and sources of variations in UV irradiance related to various bright magnetic features as a function of solar cycle. The outcome of this research effort will also be a significant improvement of the current UV irradiance models and will have a great importance in both atmospheric and space physics. This model will be an ideal platform to present and incorporate the spatially resolved research results into it. The results of the segmentation of the coronal features in relation to UV irradiance will be published elsewhere.

\section{Acknowledgments}

This research work was carried out under PROBA2 Guest Investigators Program. A part of this research work was done while R. Kariyappa was visiting Royal Observatory of Belgium in 2010 and 2011 as a Guest Investigator. The authors 
wish to express their sincere thanks to Ingolf Dammasch and Sarp Yalim, for their help in using the LYRA irradiance and SWAP full-disk integrated intensity data. Thanks to A.A Pevtsov, National Solar Observatory/Synoptic Optical Longterm Investigations of the Sun-Integrated Sunlight Spectrometer (NSO/SOLIS-ISS) for providing Ca II K parameter time series data. SOLIS data used here are produced cooperatively by National Science Foundation/National Solar Observatory (NSF/NSO) and National Aeronautics and Space Administration/Living with a Star Program (NASA/LWS). They would like to express their sincere thanks to the two referees for their critical comments and suggestions which helped to improve the paper.

\section{References}

[1] G. J. Rottman, "Observations of solar UV and EUV variability," Advances in Space Research, vol. 8, no. 7, pp. 53-66, 1988.

[2] G. Brasseur, "The response of the middle atmosphere to longterm and short-term solar variability: a two-dimensional model," Journal of Geophysical Research, vol. 982, pp. 2307923090, 1993.

[3] L. L. Hood, J. L. Jirikowic, and J. P. McCormack, "Quasidecadal variability of the stratosphere: in uence of long-term solar ultraviolet variations," Journal of Atmospheric Sciences, vol. 50, pp. 3941-3958, 1993.

[4] T. Egorova, E. Rozanov, E. Manzini et al., "Chemical and dynamical response to the 11-year variability of the solar irradiance simulated with a chemistry-climate model," Geophysical Research Letters, vol. 31, no. 6, Article ID L06119, 2004.

[5] E. Rozanov, M. Schraner, M. Wild et al., "Assessment of the Ozone and Temperature Trends for 1975-2000 with a transient Chemistry-Climate Model," in Proceedings of the 35th COSPAR Scientific Assembly, J.-P. Paillé, Ed., vol. 35, p. 2640, 2004.

[6] H. Hudson and R. Willson, "The nature of the solar-cycle variation of total irradiance," in Solar Radiative Output Variations, P. Foukal, Ed., p. 318, 1988.

[7] C. Frohlich, "Irradiance observations of the sun," in Poster Proceedings from IAU Colloquium 143: The Sun as a Variable Star: Solar and Stellar Irradiance Variations, J. M. Pap, C. Frohlich, H. S. Hudson, and S. K. Solanki, Eds., p. 28, 1994.

[8] P. Foukal and J. Lean, "Magnetic modulation of solar luminosity by photospheric activity," Astrophysical Journal, vol. 328, pp. 347-357, 1988.

[9] R. Kariyappa and J. M. Pap, "Contribution of chromospheric features to uv irradiance variability from spatially-resolved ca ii k spectroheliograms i. A New Method of Analysis and Preliminary Results," Solar Physics, vol. 167, no. 1-2, pp. 115123, 1996.

[10] J. R. Worden, O. R. White, and T. N. Woods, "Evolution of chromospheric structures derived from Ca II K spectroheliograms: implications for solar ultraviolet irradiance variability," Astrophysical Journal, vol. 496, no. 2, pp. 998-1014, 1998.

[11] J. Lean, "Solar ultraviolet irradiance variations-a review," Journal of Geophysical Research, vol. 92, pp. 839-868, 1987.

[12] H. P. Warren, J. T. Mariska, and J. Lean, "A new model of solar EUV irradiance variability 1. Model formulation," Journal of Geophysical Research A, vol. 106, no. 8, pp. 15745-15757, 2001.

[13] T. Woods, F. Eparvier, and D. Woodraska, "Solar ultraviolet variability during the TIMED mission," in Proceedings of the AGU Fall Meeting Abstracts, p. B2, San Francisco, Calif, USA, December 2003.

[14] J. L. Lean, H. P. Warren, J. T. Mariska, and J. Bishop, "A new model of solar EUV irradiance variability 2. Comparisons with empirical models and observations and implications for space weather," Journal of Geophysical Research A, vol. 108, no. 2, article no. 1059, 2003.

[15] J. F. Hochedez, W. Schmutz, Y. Stockman et al., "LYRA, a solar UV radiometer on Proba2," Advances in Space Research, vol. 37, no. 2, pp. 303-312, 2006.

[16] A. Benmoussa, I. E. Dammasch, J.-F. Hochedez et al., "Preight calibration of LYRA, the solar VUV radiometer on board PROBA2," Astronomy and Astrophysics, vol. 508, pp. 10851094, 2009.

[17] D. Berghmans, J. F. Hochedez, J. M. Defise et al., "SWAP onboard PROBA 2, a new EUV imager for solar monitoring," Advances in Space Research, vol. 38, pp. 1807-1811, 2006.

[18] J.-P. Halain, D. Berghmans, J.-M. Defise et al., "First light of SWAP on-board PROBA2," in Space Telescopes and Instrumentation 2010: Ultraviolet to Gamma Ray, vol. 7732 of Society of Photo-Optical Instrumentation Engineers (SPIE) Conference Series, July 2010.

[19] O. C. Wilson, "Flux measurements at the centers of stellar hand kLines," Astrophysical Journal, vol. 153, p. 221, 1968.

[20] R. Kariyappa and K. R. Sivaraman, "Variability of the solar chromospheric network over the solar cycle," Solar Physics, vol. 152, no. 1, pp. 139-144, 1994.

[21] R. Kariyappa, J. M. Pap, K. S. Balasubramaniam, and J. R. Kuhn, "Preliminary results of the analysis of CAII K spectroheliograms," in Helioseismology, vol. 376 of ESA Special Publication, p. 429, 1995.

[22] R. Kariyappa, "Quiet-sun variability with the solar cycle," in High Resolution Solar Physics: Theory, Observations, and Techniques, T. R. Rimmele, K. S. Balasubramaniam, and R. R. Radick, Eds., vol. 183 of Astronomical Society of the Pacific Conference Series, p. 501, 1999.

[23] R. Kariyappa, "Call K imaging to understand UV irradiance variability," Journal of Astrophysics and Astronomy, vol. 21, no. 3-4, pp. 293-297, 2000.

[24] R. Kariyappa and K. R. Sivaraman, "Variability of caII K emission flux over the solar cycle," Bulletin of the Astronomical Society of India, vol. 33, pp. 365-365, 2005.

[25] R. Kariyappa and L. Dame, "Contribution of chromospheric features to UV irradiance variability," in Proceedings of the IAU Joint Discussion, vol. 8, August 2006.

[26] M. K. V. Bappu and K. R. Sivaraman, "K emission-line widths and the solar chromosphere," Solar Physics, vol. 17, no. 2, pp. 316-330, 1971. 

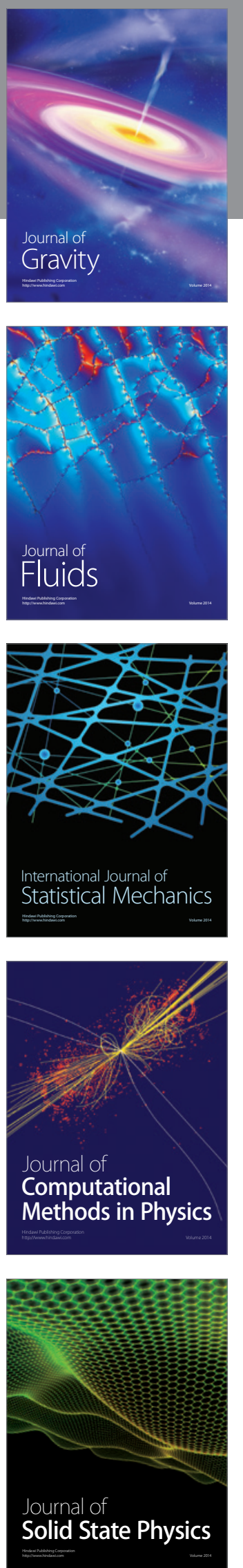

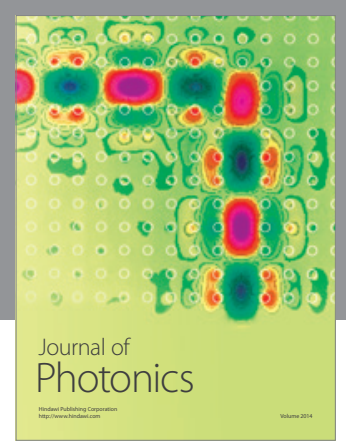

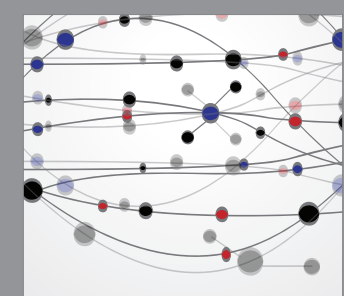

The Scientific World Journal
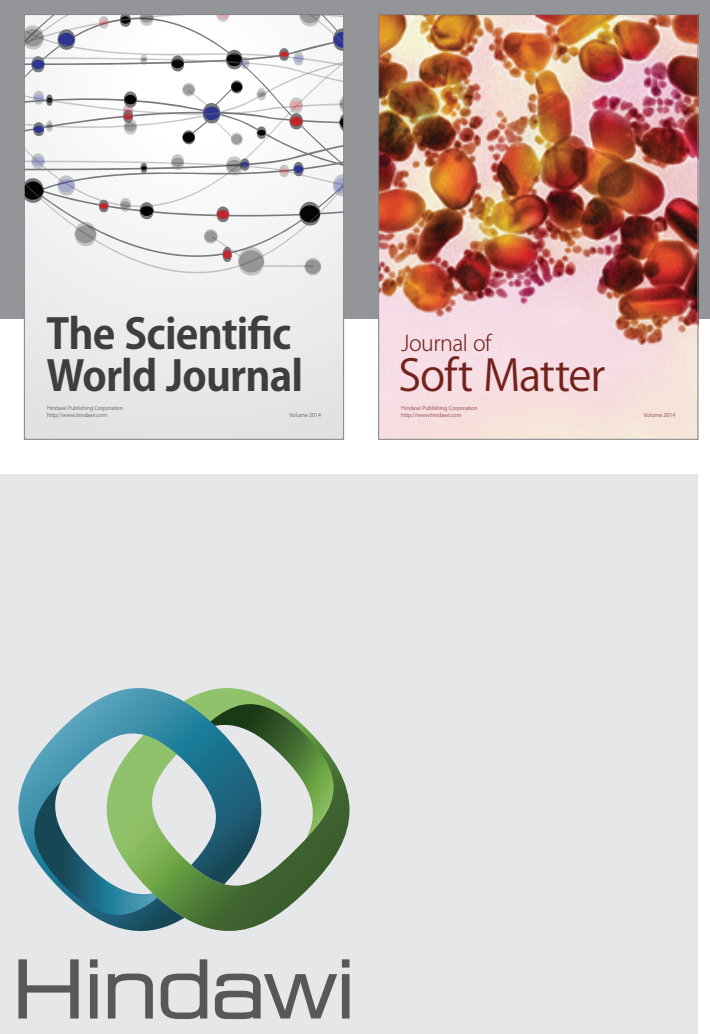

Submit your manuscripts at

http://www.hindawi.com
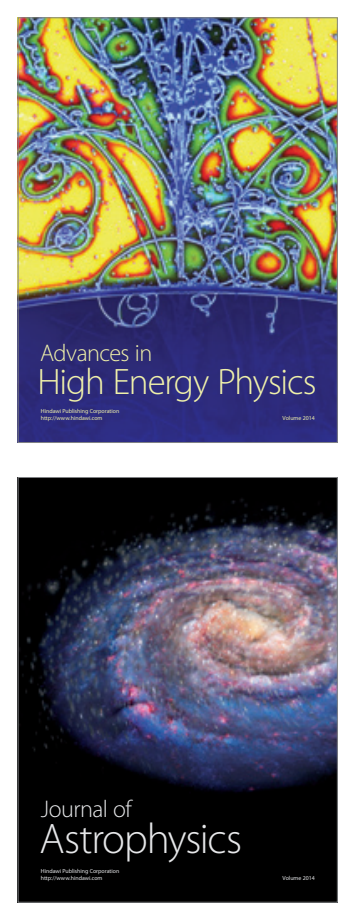
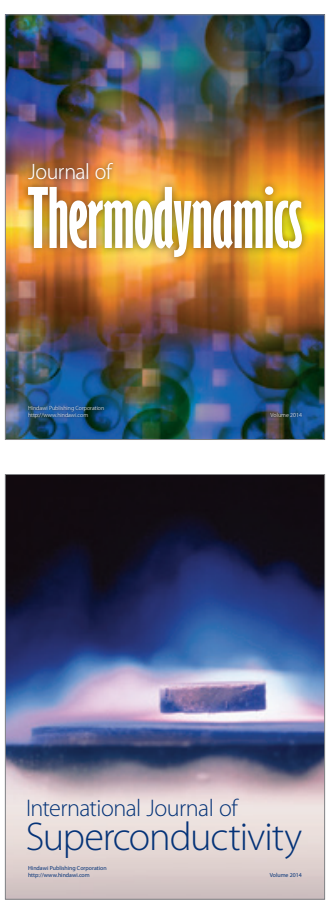
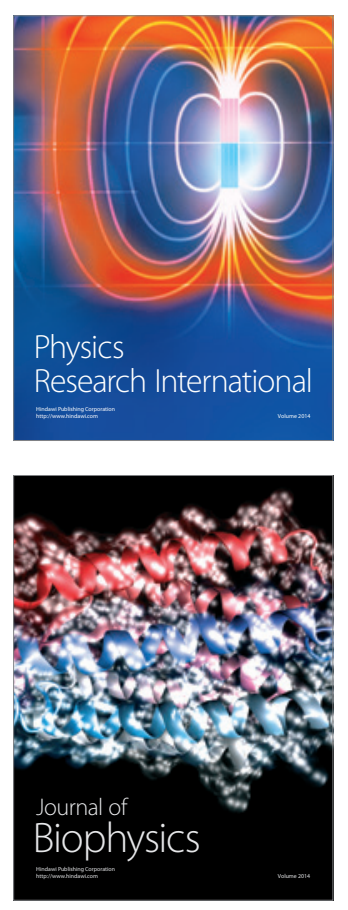
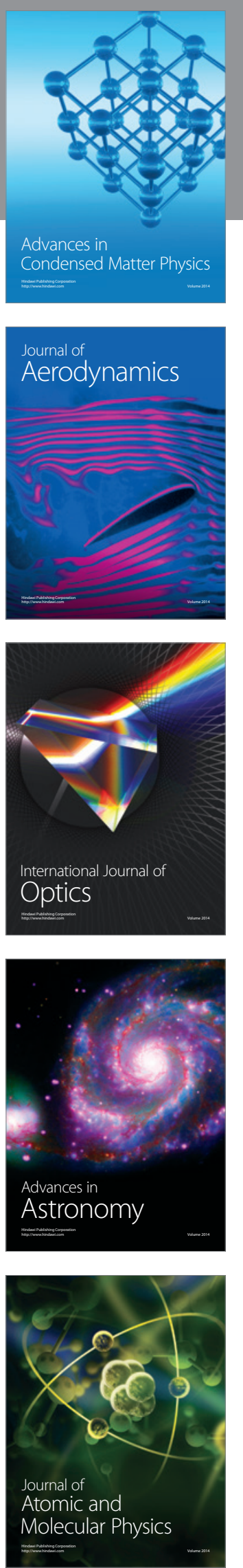